\title{
Integrated application of multi-omics strategies provides insights into the environmental hypoxia response in Pelteobagrus vachelli muscle
}

\author{
Jie Li $^{1}$, Xia Liang ${ }^{1}$, Jiejie Xu ${ }^{1}$, Jiajia Zhang ${ }^{1}$, Kai Zhang ${ }^{1}$, Jie Ji ${ }^{1}$, Tao Wang ${ }^{1}$, Yongyi Jia ${ }^{1}$, \\ Guosong Zhang ${ }^{1}$, and Shaowu Yin ${ }^{2}$ \\ ${ }^{1}$ Affiliation not available \\ ${ }^{2}$ Nanjing Normal University
}

February 7, 2021

\begin{abstract}
Abstract Increasing pressures on aquatic ecosystems due to pollutants, nutrient enrichment and global warming have severely depleted oxygen concentrations. This sudden and significant lack of oxygen has resulted in persistent increases fish mortality rates. Revealing the molecular mechanism of fish hypoxia adaptation will help researchers to find hypoxic markers for hypoxia induced by environmental stress. Here, we used a multi-omics approach to identify several hypoxia-associated miRNAs, mRNAs, proteins, and metabolites involved in diverse biological pathways in the muscles of Pelteobagrus vachelli. Our findings revealed significant hypoxia-associated changes in muscles over $4 \mathrm{~h}$ of hypoxia exposure and discrete tissue-specific patterns. We have previously reported that P. vachelli livers exhibit increased anaerobic glycolysis, heme synthesis, erythropoiesis, and inhibit apoptosis when exposed to hypoxia $4 \mathrm{~h}$. However, the opposite was observed in muscles. According to our comprehensive analysis, fishes show an acute response to hypoxia, including activation of catabolic pathways to generate more energy, reduction of biosynthesis to decrease energy consumption, and shifting from aerobic to anaerobic metabolic contributions. Also we found that hypoxia induced muscle dysfunction by impairing mitochondrial function, activating inflammasomes, and apoptosis. The hypoxia-induced mitochondrial dysfunction enhanced oxidative stress, apoptosis, and further triggered IL- $1 \beta$ production via inflammasome activation. In turn, IL-1 $\beta$ further impaired mitochondrial function or apoptosis by suppressing downstream mitochondrial biosynthesis-related proteins, thus resulting in a vicious cycle of inflammasome activation and mitochondrial dysfunction. Our findings contribute meaningful insights into the molecular mechanisms of hypoxia, and the methods and study design can be utilized across different fish species.
\end{abstract}

\section{Introduction}

Aerobic animals use oxygen to generate energy through oxidative phosphorylation within mitochondria. Currently, aquatic ecosystems are increasingly under pressure from pollutants, nutrient enrichment, and global warming, all of which more increase the risk of hypoxia in water (Mandicet al. 2015). Therefore, aquatic organisms are usually exposed to different concentrations of dissolved oxygen. A severe and sudden hypoxia can be fatal for hypoxia-sensitive fish. Revealing the molecular mechanism of fish hypoxia adaptation will help us find the hypoxic marker caused by environmental stress (Abdelrahman et al. 2017).

In order to maintain organism function and homeostasis in a hypoxic environment, aquatic organisms, including fishes, respond by various behavioral and physiological adjustments, e.g., decreasing swimming rate, increasing respiration rate, increasing oxygen supply, and reducing oxygen consumption (Sappalet al. 2016; Zhong et al.2009). These process depends on the division and cooperation among tissues in fishes, such as liver, muscle, brain, heart, gill. Relevant research results show that there are differences in the sensitivity and tolerance of different tissues, indicating that the tissue-specific expression pattern reflects the different 
metabolic effects of tissues during hypoxia (Zhu et al. 2013). For example, fish brain and heart showed fewer examples of down-regulation compared with muscle, in keeping with the need for sustained activity (Gracey et al. 2001; Lardon et al. 2013). The different patterns may also be related to the extent of the hypoxic insult that each tissue experiences. For example, fish heart receives blood directly from the gills and thus will have a better oxygen supply than other tissues (Everett et al. 2012; Ju et al. 2007).

Now, various methods in fish were applied for studies of the hypoxia molecular mechanisms, e.g., investigations for transcriptional changes using qRT-PCR (Chen et al. 2012; Wang et al. 2015), transcriptome (Beck et al. 2016; Gong et al. 2020; Yang et al. 2018), and changes in protein using 2D-DIGE (Dowd et al.2010; Smith et al. 2009; Wulff et al. 2012). Biological phenomena induced by environmental stress are changeable and complex, which is also accompanied by complicated regulation of miRNA/gene/protein expression. Multiple omics are combination of two or more omics methods, and can avoid the incomprehensive conclusions of single-omics (Sun et al. 2018). Unfortunately, the study of the fishes responding mechanism undergo hypoxia using multi-omics is negligible, which vastly hinders the comprehensive understanding of this biological phenomenon.

Pelteobagrus vachelli has become a popular commercial fishes in Asia because of its relatively high yield and an affordable price for consumers (Guosong et al. 2017). However, due to its low oxygen threshold and high oxygen consumption rate, this species is only found in the rivers. A sudden lack of oxygen can result in mortality and finally cause the pond turnover (Zhang et al. 2016a). These properties indicate that $P$. vachelli is not only an important aquaculture variety, but also a potential model fishes for studying the molecular mechanisms of hypoxia. Recently, the miRNA-mRNA regulatory network has been shown to respond to hypoxia in livers ofMegalobrama amblycephala (Sun et al. 2017) and P. vachelli (Zhanget al. 2016b). This suggests that the HIF-1 signaling may be involved in fish hypoxic molecular adaptation, which is often regulated and controlled by the physiological changes. Also, we conducted iTRAQ proteomic analysis in $P$. vachelli livers to uncover hypoxic responsive proteins involved in diverse biological pathways, e.g., peroxisome, glycolysis, PPAR signaling, lipid and amino acid metabolism (Zhang et al. 2017).

At present, relative to other tissues, studies on the mechanism of fish muscle, the largest tissue, response to hypoxia are relatively scarce. Therefore, we characterize transcriptomic, miRNAomic, proteomic and metabolomic changes of $P$. vachellimuscles under environmental hypoxia simultaneously (Fig. 1 ), combined with the previous studies on $P$. vachelli liver. Our findings aim to offer deeper insights into tissue-specific patterns of expression induced by environmental hypoxia, on the other hand the methods and study design can be used to analyze markers to profile other species undergo hypoxic environmental stress.

Fig. 1. General workflow of bioinformatics analysis conducted to investigate the molecular mechanism of hypoxia adaptation in $P$. vachelli in terms of 4 main aspects (changes in posttranscriptional, transcriptional, protein, and metabolite levels). 


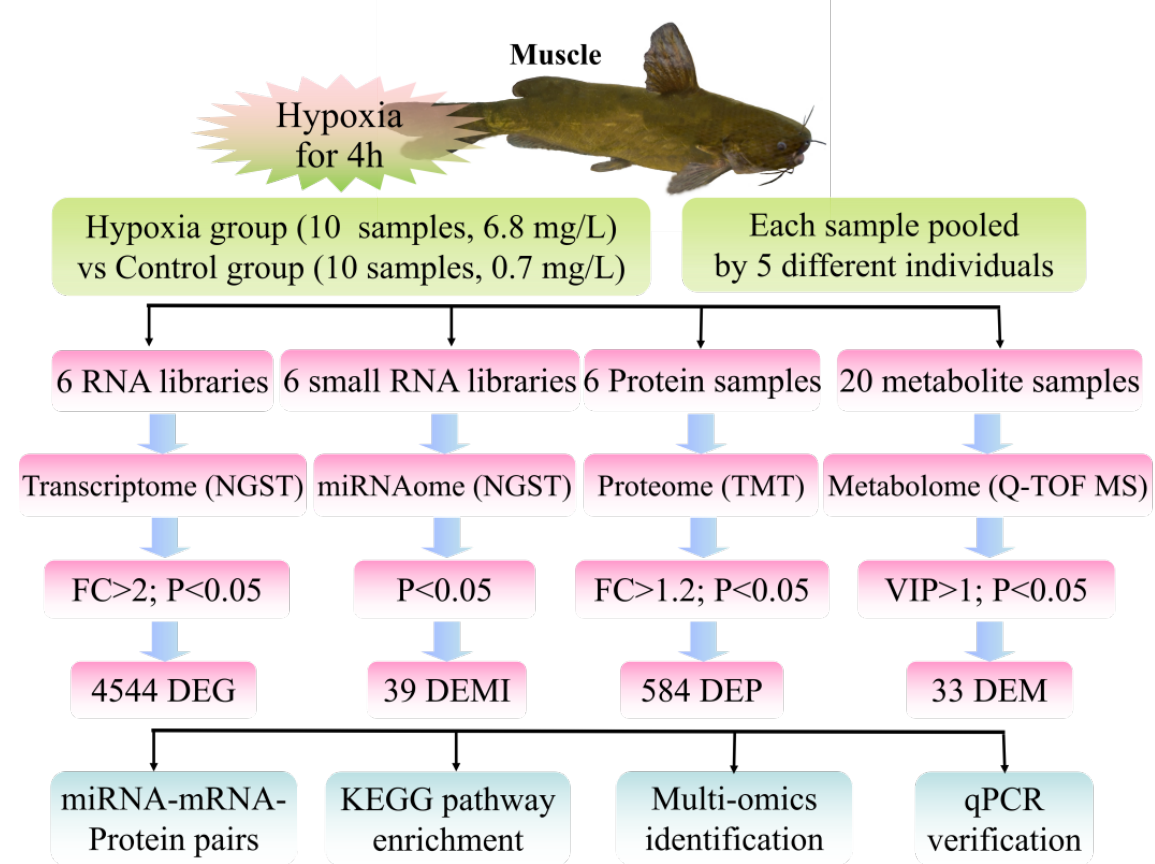

\section{Materials and methods}

\subsection{Experimental design}

First, $240 P$. vachelli $(14 \pm 1.25 \mathrm{~cm}$ in length, $22 \pm 1.78 \mathrm{~g}$ in weight) were acclimated for three weeks. They were sequentially feed restricted for two days in an aquarium (volume of $600 \mathrm{~L} ; 26 \pm 1^{\circ} \mathrm{C}$ ). All the experimental methods were the same as description in our previous article (Zhang et al.2016b). In order to reduce the sequencing error caused by the sample itself, the muscle tissue was all taken from the same spot of the fish. Each control sample (No. M0a, M0b, M0c, M0d, M0e, M0f, M0g, M0h, M0i, M0j; 6.8 $\mathrm{mg} / \mathrm{L}$ ) was made up of five different individual muscle from a total 50 control fish. Afterwards the dissolved oxygen in water from $6.8 \mathrm{mg} / \mathrm{L}$ to $0.7 \mathrm{mg} / \mathrm{L}$ was reduced by bubbling pure nitrogen by 30-35 min. After dissolved oxygen was maintained for $4 \mathrm{~h}$ at $0.7 \mathrm{mg} / \mathrm{L}, 50$ treated fish (No. M4a, M4b, M4c, M4d, M4e, M4f, M4g, M4h, M4i, M4j; $0.7 \mathrm{mg} / \mathrm{L}$ ) were rapidly acquired for muscle dissection. Control and treated samplings had 10 biological replicates respectively, which were used to conduct metabolome analysis. Meanwhile, six of the same harvested samples (No. M0a, M0b, M0c, M4a, M4b, M4c) were used to conduct analyses of transcriptome, miRNAome, proteome, and qRT-PCR.

\subsection{Differentially expressed genes (DEG) analysis}

mRNA-seq method was the same as description in our previous article (Zhanget al. 2019), including RNA extraction, six cDNA libraries construction, illumina Hiseq 4000 sequencing, de novo assembly, unigene annotation and functional classification (Patro et al.2017). The expression level for unigenes were calculated by TPM using Salmon (Mortazavi et al. 2008). DEG were selected with $P<0.05$ and fold change $>2$ using R package edgeR (Robinson et al. 2010). KEGG pathway enrichment analysis of DEG was the same as description in our previous article, and so as DEP and DEM (Zhang et al. 2019).

\subsection{Differentially expressed miRNAs (DEMI) analysis}

miRNA-seq method was the same as description in our previous article, including six small RNA libraries construction, illumina Hiseq 4000 sequencing, miRNA identification, prediction of target genes of miRNAs (Zhang et al. 2019). DEMI based on standardized deep-sequencing counts was analyzed by Student's t-test $(P<0.05)$ (Li et al. 2017; Zhao et al. 2017). 


\subsection{Differentially expressed proteins (DEP) analysis}

TMT-based quantitative proteomic analysis was the same as description in our previous article, including Protein extraction and digestion, protein digestion, TMT labeling, low-pH nano-HPLC-MS/MS analysis, and the database searching (Zhang et al. 2019). Database searching was conducted using the present RNA-seq results (Accession codes: SUB7765255) by Mascot.

For protein quantitation, a protein was required to contain at least two unique peptides. 9 protein ratios were produced by comparing $3 \mathrm{M} 4$ and $3 \mathrm{M} 0$ (i.e., M4a/M0a, M4a/M0b, M4a/M0c, M4b/M0a, M4b/M0b, M4b/M0c, M4c/M0a, M4c/M0b, M4c/M0c). then the P values were determined using ANOVA (Student's t-test) and the 9 ratios. The mean comparisons of pairs among the 9 protein ratios calculated fold changes. Finally proteins with $P<0.05$ and fold change $>1.2$ were considered to be DEP (Chen et al.2013b; Zhang et al. 2019).

\subsection{Differentially expressed metabolites (DEM) analysis}

Metabolome method was the same as description in a previous study, including 20 metabolite extraction (No. M0a, M0b, M0c, M0d, M0e, M0f, M0g, M0h, M0i, M0j, M4a, M4b, M4c, M4d, M4e, M4f, M4g, M4h, M4i, M4j), LC-MS analysis conditions, metabolomics data processing (Cao et al. 2020). Data analysis methods include PLS-DA and PCA. Quantitative identification of metabolites screening and supervised PLS-DA were evaluated by MetaX software (Cao et al. 2020). The VIP was calculated, and metabolites with VIP > 1 for both multidimensional statistical analysis and $P<0.05$ (Student's t-test) were selected as metabolites with significant differences (Wen et al. 2019).

\subsection{Multi-omics analysis of transcriptome, miRNAome, proteome, and metabolome}

ACGT101-CORR 1.1 (LC Sciences, USA) was used to construct the negative miRNA-mRNA regulatory network (up-down and down-up). In brief, transcriptome and miRNAome were integrated by combining the expression profiles of DEG and DEMI with miRNA-targeting information. Afterwards the protein informations added miRNA-mRNA pairs to the miRNA-mRNA-protein pairs (up-down-down and down-up-up) directly. Secondly, 145 miRNA-mRNA-protein pairs and 33 DEM were combined to recognite mutual KEGG pathways. Finally, cytoscape software was mapped to generate an interaction network among the relationships of DEMI, DEG/DEP, DEM (Wenet al. 2019).

\section{7 qRT-PCR}

The expression profiles of all 16 DEMI among the multi-omics interaction network and the selected 70 DEG representing biological processes induced by hypoxia were further analyzed using qRT-PCR. qRT-PCR methods were the same as description in our previous article (Zhang et al. 2019). All primers are shown in Table S1 . $2^{-\mathrm{T}}$ method was used to calculate the expression levels, and the data were subjected to statistical significant analysis $(P<0.05)$ using SPSS 22.0 (Student's t-test).

\section{Results}

\subsection{Transcriptome summary}

A total of 28,696 unique genes had been identified from six cDNA libraries (M0a, M0b, M0c, M4a, M4b, and M4c) representing $P$. vachelli muscles (Table S2 ). The quality control analysis of the transcriptome is shown in Table S3, including sequencing quality control overview, trinity assembly results overview, gene/transcript length overview, and gene function checklist. We found that 3511 genes were significantly upregulated, whereas 1033 genes were significantly downregulated in response to hypoxia $(P<0.05$ and Fold change $>2$ ). By performing the KEGG pathway analyses for 4544 differentially expressed genes (DEG), a total of 22 pathways were identified that showed significant changes after hypoxia $(\mathrm{P}<0.05)($ Fig. 2A $)$.

Among these pathways (Fig. 2A ), the "VEGF signaling pathway" involved in mediating vasopermeability, angiogenesis, and vascular tone was first enriched. However, DEG of "African trypanosomiasis" reflected the biological process of anemia (hemoglobin synthesis, down-regulated). Afterwards catabolism and anabolism 
were the two most represented subclasses. In addition, some important subclasses have also been significantly enriched, including human immune and inflammation. Finally, we found that "DCM" and "HCM" represented the impaired muscle function, which were also significantly enriched $(P<0.05)$. Interestingly, the numbers of upregulated genes were generally greater than that of downregulation for biological process of vessel, catabolism, immune, inflammation, muscle dysfunction, and vice versa for anemia and anabolism.

Fig. 2. KEGG pathway enrichment of 4544 differentially expressed genes (DEG; A), 584 differentially expressed proteins (DEP; B) and 33 differentially expressed metabolites (DEM; C) $(22,14,14$ pathways; $P<0.05)$.
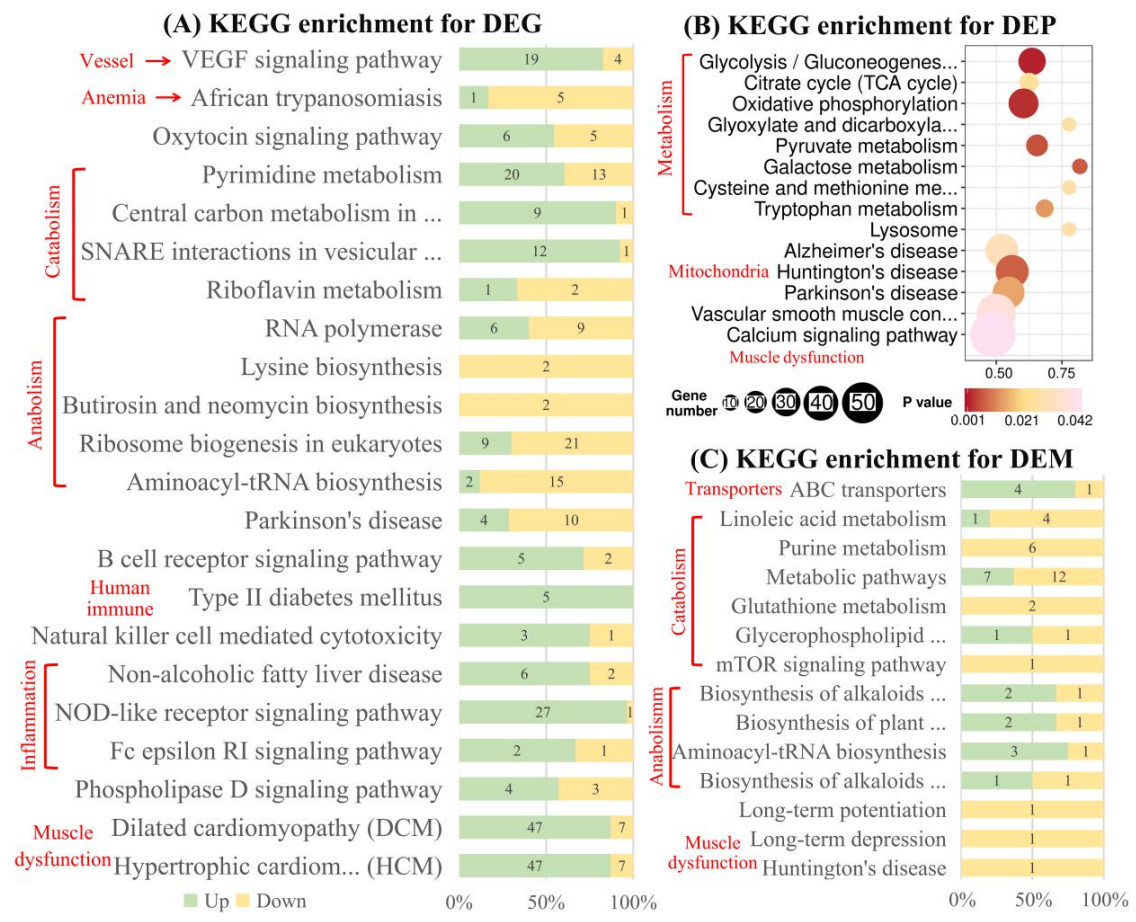

\subsection{Proteome summary}

All MS/MS spectra were processed using Mascot from six protein samples (M0a, M0b, M0c, M4a, M4b, and M4c). TMT analysis of $P$. vachellimuscle proteome showed 14,943 unique peptides from 41,278 unique spectra in this database $(334,625$ total spectra) and resulted in 1,551 unique proteins. All peptide/protein identifications and quantifications are shown in Table S2 . The quality control analysis of the proteome is shown in Table S3, including mass delta, the distribution diagram of peptide number, peptide length, protein cleavages, and protein mass. All coefficients of variation distribution for proteome showed that a total of $97.36 \%$ of the identified proteins displayed a ratio \% CV $<20 \%$, and proteome project has a good repeatability (Table S3).

A total of 584 differentially expressed proteins ( DEP; fold change $>1.2$ and $P<0.05$ ) were reliably quantified using TMT analysis, including 9 upregulated and 575 downregulated proteins undergo hypoxia. KEGG enrichment analysis $(P<0.05)$ displayed a total of 14 significantly enriched pathways under hypoxia (Fig. 2B ). Muscle function, amino acid and carbohydrate metabolism were the three most represented subclasses. In addition "Alzheimer's disease", "Huntington's disease", "Parkinson's disease" containing mitochondrial related proteins was significantly enriched by down DEP. Of note, DEP of carbohydrate metabolism referred to aerobic metabolism mainly centered on "Pyruvate metabolism" (e.g., CS, IDH, DLD, OGDH, SDHa/b, 
MDH1, down-regulated) and the DEP of amino acid metabolism and human disease involved in amino acid synthesis and muscle dysfunction.

Fig. 3. KEGG pathway enrichment analyses of 1809 negative miRNA-mRNA pairs (A) and 145 negative miRNA-mRNA-protein pairs $(B)(20$ and 12 pathways; $P<0.05)$.
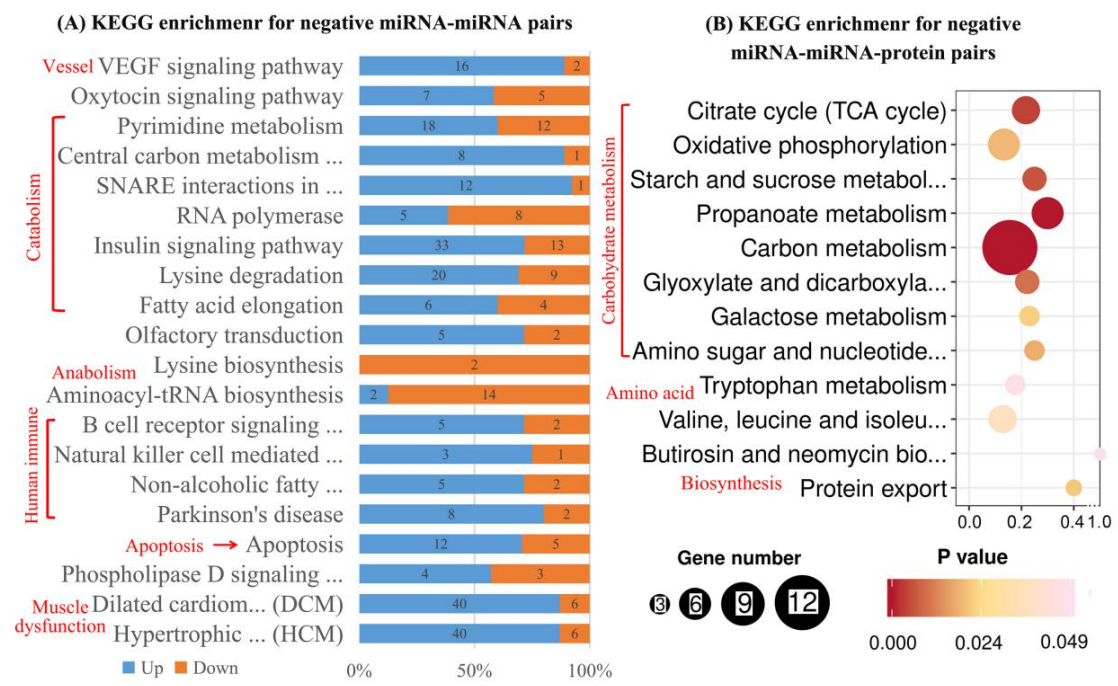

\subsection{Correlation of miRNA-mRNA-protein regulatory network}

348 conserved miRNAs belonging to 292 miRNA seed-based families were identified in the six small RNA libraries (M0a, M0b, M0c, M4a, M4b, and M4c). The quality control analysis of the miRNAome is shown in Table S3, including overview of reads from raw data to cleaned sequences, summary of known and predicted miRNA, length distribution of sequences, and conservation of the identified miRNA with other species. A total of 39 differentially expressed miRNAs (DEMI) were found $(P<0.05)$, with 17 upregulated and 22 downregulated miRNAs (Table S2 ).

In our results, according to the DEMI, DEG and DEP datasets and miRNA-targeting information, 1809 negative miRNA-mRNA regulatory network with the expression pattern of up-down and down-up were detected (Table S4 ). Further, 12 of 20 significant enriched KEGG pathways for 1809 pairs were found to be overlapping for those of transcriptome, also involved in vessel formation, metabolism, immune response and muscle dysfunction. Additionally, similar to the DEG, the numbers of upregulated genes were greater than that of downregulated ones involved in vessel formation, catabolism, immune response, muscle dysfunction, and vice versa for anabolism (Fig. 3A ). Finally we identified 145 negative miRNA-mRNA-protein pairs (up-down-down and down-up-up) with negative correlation as the key for analysis including 21 DEMI and 60 co-DEG/DEP in total (Fig. 4A ). Moreover, five out of 11 significant enriched KEGG pathways for 145 pairs were found to be overlapping for those of proteome, such as "Citrate cycle (TCA cycle)", "Oxidative phosphorylation", "Tryptophan metabolism", "Glyoxylate and dicarboxylate metabolism", and "Galactose metabolism", with carbohydrate metabolism of the most commonly represented subclasses. In addition, biosynthesis related to "Butirosin and neomycin biosynthesis" and "Protein export" were significantly enriched by down co-DEG/DEP (Fig. 3B ).

\subsection{Metabolome summary}

A total of 9433 metabolic ion peaks were extracted from $P$. vachelli muscles in the control group (M0a, M0b, M0c, M0d, M0e, M0f, M0g, M0h, M0i, M0j) and those in the experimental group (M4a, M4b, M4c, M4d, M4e, M4f, M4g, M4h, M4i, M4j), including 5152 positive and 4281 negative ion peaks (Table S2 ). 
In addition we found that 33 unique differentially expressed metabolites (DEM with MS2; 14 up-regulated and 19 down-regulated) were among the 18 positive and 24 negative DEM (VIP $>1$ and $P<0.05$; Table S2).

The PCA model showed that the positive and negative ion modes were closely clustered. The PLS-DA model of the example set was also established. The model evaluation parameters with positive ion mode $(\mathrm{R} 2=0.99 \mathrm{cum}, \mathrm{Q} 2=0.78 \mathrm{cum})$ and negative ion mode $(\mathrm{R} 2=0.99 \mathrm{cum}, \mathrm{Q} 2=0.87 \mathrm{cum})$. Above PCA and PLS-DA results indicated that the model was reliable and stable (Table S3 ). Similar to the KEGG analysis of DEG, 14 significant pathways for the biological process of catabolism, anabolism, and muscle dysfunction were enriched by $33 \mathrm{DEM}(P<0.05)$. Significant decreases in intermediate metabolites were found in catabolism, however, the upregulated intermediate metabolites were a big part among anabolic pathways (Fig. 2C).

Fig. 4. 145 negative miRNA-mRNA-protein pairs including 21 DEMI and 60 co-DEG/DEP (A) and multi-omics interaction network including 16 DEMI, 23 co-DEG/DEP and 17 DEM (B).
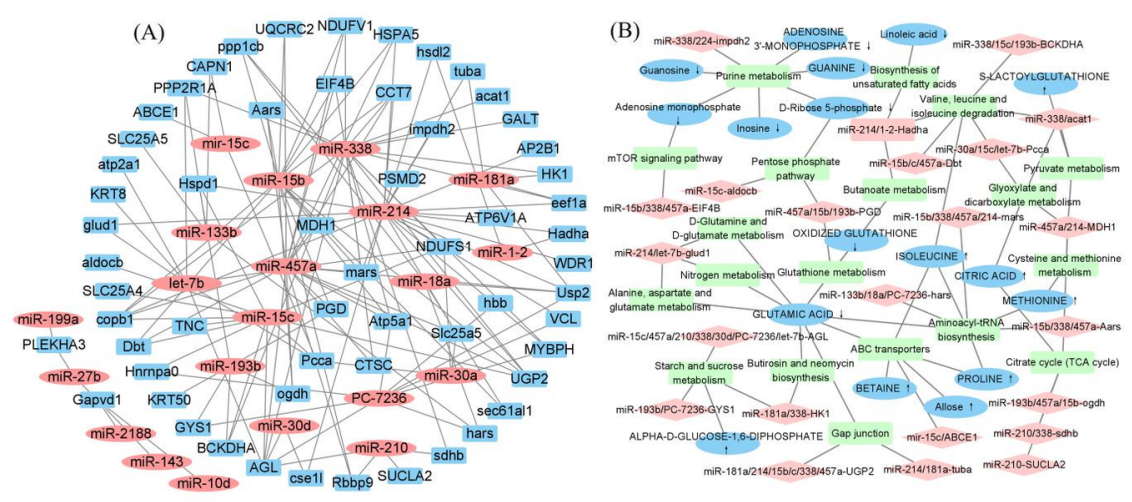

\subsection{Multi-omics identification of key miRNAs, genes, proteins, and metabolites}

The hierarchical clustering of the DEP, DEMI, DEG and DEM based on the fold change' $\mathrm{Z}$ score of M4a/(M4b, M4c, M0a, M0b, M0c), six samples' log10 (Norm), six samples' Z score of TPM and 20 samples' $\mathrm{Z}$ score of Norm were showed that all samples can be divided into two different groups according to control and hypoxia treatment (Fig. S1) . In general, environmental hypoxia had a significant effect on the overall gene /miRNA/ protein/metabolite expression profile of $P$. vachellimuscle.

In this study, the transcriptome, miRNAome, proteome and metabolome of $P$. vachelli muscle under hypoxia stress were integrated by investigating whether changes in "genes/proteins" levels correlated with changes in the corresponding "negative miRNAs", and coupled with mutual KEGG pathways for DEM. This was based on 145 negative miRNA-mRNA-protein pairs and 33 unique DEM, 16 DEMI, 23 co-DEG/DEP and 17 DEM were involved in the 19 mutual KEGG pathways (Fig. 4B ).

These mutual pathways contained carbohydrate metabolism (i.e., TCA cycle, pentose phosphate pathway, starch, sucrose, pyruvate, butanoate, glyoxylate and dicarboxylate metabolism), amino acid metabolism (i.e., valine, leucine, isoleucine, cysteine, methionine, alanine, aspartate, glutamate, D-Glutamine and Dglutamate metabolism), other metabolic processes (i.e., purine and nitrogen metabolism, biosynthesis of aminoacyl-tRNA, unsaturated fatty acids, butirosin and neomycin), membrane transport (i.e., ABC transporters), signal transduction (i.e., mTOR signaling pathway), and cellular community (i.e., gap junction). Genes produce proteins through complex transcription and translation processes to regulate the metabolism of organisms (Table S5 ). In our case, downregulated miR-193b/457a/15b-OGDH, miR-210/338-SDHb, and 
miR-457a/214-MDH1 led to suppression of aerobic metabolism (e.g., "Pyruvate metabolism" and "TCA cycle"), thus making intermediate products rise (e.g., citric acid and S-lactoylglutathione). "Aminoacyl-tRNA biosynthesis" was regulated by downregulated miR-15b/338/457a-Aars/mars and miR-133b/18a/PC-7236hars to accumulate intermediates (proline, isoleucine, and methionine).

Fig. 5. Relative miRNA expression of all 16 DEMI among the multi-omics identification for comparison of the M4 versus M0 groups, with respect to qRT-PCR and miRNA-seq.

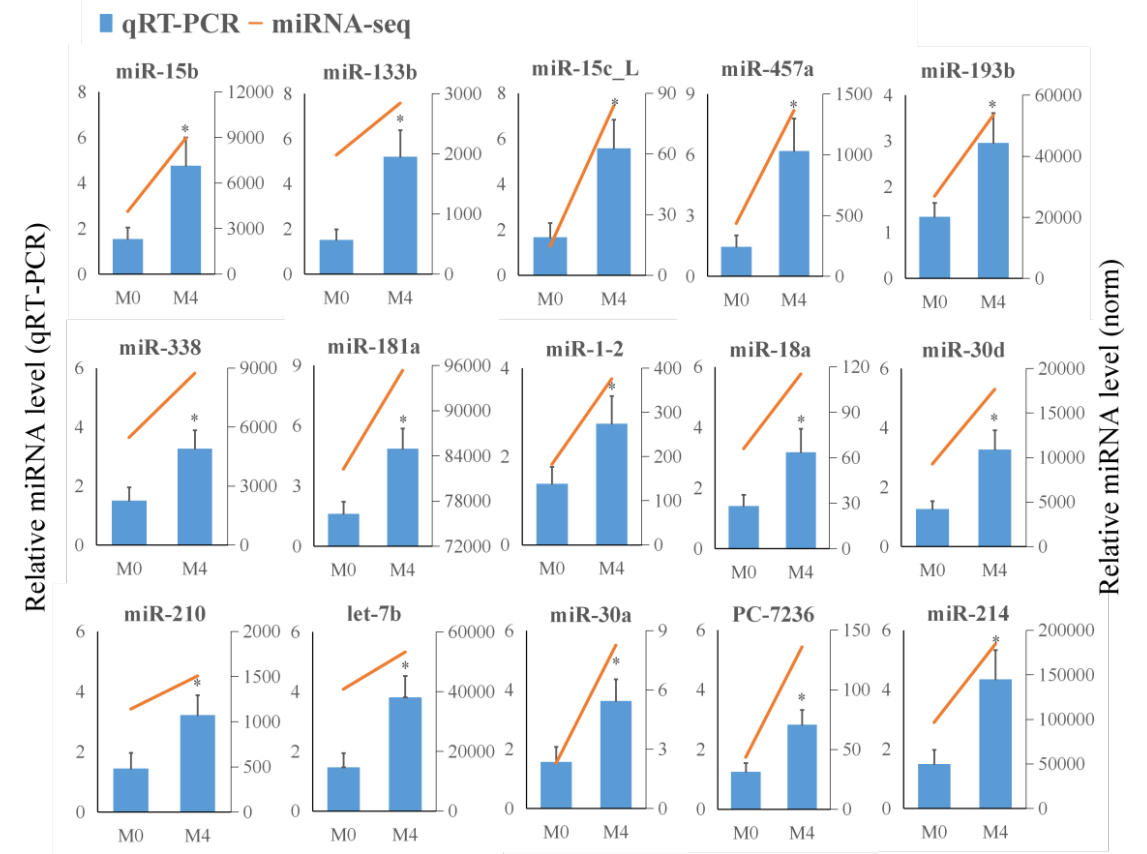

\section{6 qRT-PCR analysis}

To further analyze the hypoxia-induced biological processes in $P$. vachelli muscles, we performed qRT-PCR analysis on selected 16 DEMI and 70 DEG. These representative miRNAs/genes were artificially selected to determine their potential role in environmental hypoxia for P. vachelli muscles. For example, all 16 DEMI (miR-15b/c, 133b, 181a, 193b, 338, 457a, 1-2, 18a, 30a/d, 210, 214, PC-7236, let-7b) were from the multiomics identification (Fig. 5 ) and some DEGs were related to important biological processes (i.e., oxygen sensor, angiogenesis, heme and erythropoiesis, glycolysis, TCA cycle, lipolysis, oxidative stress, mitochondrial dysfunction, NOD-like receptor, NF-kappa B, MAPK signaling pathway, cell cycle, and apoptosis). The results of qRT-PCR revealed similar expression patterns between most of these mRNAs/miRNAs and those from transcriptome/miRNAome data (TPM/reads-based expression values) (Table 1; Fig. 5; Fig. 6 ). Of these, miR-15c showed minimal expression in control groups (M0a, M0b, M0c) in the qRT-PCR analysis, and was induced by hypoxia stress, which was consistent with the present miRNA-seq data. Although there were some quantitative differences between the two analysis platforms, our results showed similar trends between the qRT-PCR and RNA-seq data.

Table 1 Relative mRNA expression of 48 selected DEG for comparison of the M4 versus M0 groups, with respect to mRNA-seq and qRT-PCR.

\begin{tabular}{llll}
\hline Annotation & mRNA-seq $(\log 2$ FC) & mRNA-seq $(\log 2$ FC) & Regulation \\
\hline Oxygen sensor & Oxygen sensor & Oxygen sensor & Oxygen sensor \\
FIH & 2.26 & 2.26 & up
\end{tabular}




\begin{tabular}{llll}
\hline Annotation & mRNA-seq $(\log 2 \mathbf{F C})$ & mRNA-seq $(\log 2 \mathbf{F C})$ & Regulation \\
\hline PHD2 & 2.17 & 2.17 & up \\
PHD3 & 3.45 & 3.45 & up \\
Angiogenesis & Angiogenesis & Angiogenesis & Angiogenesis \\
VEGF & 2.49 & 2.49 & up \\
ANGPTL4 & 1.12 & 1.12 & up \\
Heme and erythropoiesis & Heme and erythropoiesis & Heme and erythropoiesis & Heme and erythropoiesis \\
ALAS2 & -1.99 & -1.99 & down \\
TF & -4.79 & -4.79 & down \\
TFRC & -1.14 & -1.14 & down \\
CA & -1.51 & -1.51 & down \\
HB $\alpha$ & -1.56 & -1.56 & down \\
HB $\beta$ & -1.53 & -1.53 & down \\
Glycolysis & Glycolysis & Glycolysis & Glycolysis \\
GLUT1 & 1.32 & 1.32 & up \\
PK & -1.05 & -1.05 & down \\
HK1 & -1.29 & -1.29 & down \\
TCA cycle & TCA cycle & TCA cycle & TCA cycle \\
PDK & 1.88 & 1.88 & up \\
PDHA & -1.03 & -1.03 & down \\
ogdh & -1.27 & -1.27 & down \\
sdhb & -1.16 & -1.16 & down \\
sdha & -1.28 & -1.28 & down \\
MDH1 & -1.06 & -1.06 & down \\
Lipolysis & Lipolysis & Lipolysis & Lipolysis \\
PPAR $\alpha$ & 1.21 & 1.21 & up \\
PPAR $\beta$ & 2.38 & 2.38 & up \\
Oxidative stress & Oxidative stress & Oxidative stress & Oxidative stress \\
Mn-SOD & Mn-SOD & 1.33 & up \\
\hline & & & \\
\hline
\end{tabular}

*Asterisk indicates statistical significance of differential gene expression with $P<0.05$ (t-test). Fold change $=\mathrm{M} 4$ group $($ mean) $/ \mathrm{M} 0$ group (mean).

\section{Discussion}

We constructed 145 negative miRNA-mRNA-protein pairs in P. vachelli muscle induced by hypoxia (Table S4), and later discovered that 16 DEMI, 23 DEG/DEP and 17 DEM were involved in 19 mutual KEGG pathways (Table S5) . The predicted miRNA-mRNA-protein-metabolite regulatory networks were more complex than previously thought. A single miRNA could regulate multi-target mRNAs/proteins/metabolite and vice versa (Fig. 4 ). In performing KEGG pathway analyses for DEG/DEP and multi-omics pairs, "signal transduction", "metabolism" and "muscle function" were found to be the three most frequently represented subclasses (Fig. 2 and Fig. 3 ). With regard to the fish hypoxia adaptation strategy, we highlighted our particular research using pathway analysis related to the functional clusters: 1. HIF-1 signaling pathway; 2. Energy metabolism; 3. Muscle function. Some DEG and their regulating miRNAs among above clusters representing hypoxic markers were further analyzed using qRT-PCR (Table 1, Fig. 5and Fig. 6 ).

\subsection{HIF-1 signaling pathway}

"HIF-1 signaling" is a major regulator of the hypoxia signaling pathway, leading to similar biochemical and physical reactions, including oxygen sensing, increasing oxygen delivery and reducing oxygen consumption (Zhu et al. 2013). 


\section{(1) Oxygen sensor}

In recent years, the relationship between several oxygen sensors and hypoxia in some fishes has been elucidated (Geng et al. 2014; Wang et al. 2015). We found that the transcriptional expressions of the oxygen sensors (FIH-1, PHD2, and PHD3) were significantly upregulated and their regulating miRNAs were significantly downregulated (Table $\mathbf{1}$ ), which indicates that oxygen sensors and their regulation of miRNAs play an vital role in response to hypoxia. Similar results were found in P. vachelliliver and brain(Guosong et al.2017).

(2) Increase oxygen delivery

The pattern of gene upregulation (19/23) in the "VEGF signaling pathway" may be involved in promoting their vasopermeability, angiogenesis (VEGF, KDR1, ANGPTL4, up-regulated) and mediating the vascular tone (NOS2, ECE1, up-regulated) (Table 1 ). Similar activation of the VEGF or ANGPTL4 has been shown in P. vachelliliver and Astronotus ocellatus(Baptista et al. 2016; Zhang et al. 2016b).

It has been widely accepted that many blood regulations in fishes contribute to improve oxygen uptake and transport to tissues in low oxygen environments. In P. vachelli similar raised blood indices (RBC, $\mathrm{HB}$ and SI/TIBC) and liver mRNA levels (TFRC, CA and EPO) have been reported (Guosonget al. 2017). However, in this study "African trypanosomiasis" was enriched by a downregulated anemia related gene. We found that the genes or proteins involved in heme synthesis (ALAS2, HRG-1A, urod, Blvra, UGT2A1, PPOX, HMOX2) and erythropoiesis (TF, TFRC, CA, HB $\alpha, \mathrm{PC}-7236-\mathrm{HB} \beta$ ) were significantly reduced (Table 1 and Fig. 5 ). This may be because the muscle tissue is not the hematopoietic organ of the fish. However, among the more highly upregulated proteins in the D. rerio muscle during hypoxia were two variants of $\mathrm{HB} \alpha$, which may be due to different period and level of hypoxia or various regulatory mechanism in different fish muscle (Chen et al. 2013a).

\section{(3) Reduce oxygen consumption}

In this study, upregulation of GLUT1 (HIF-1 $\alpha$ target gene) suggested a promotion in anaerobic metabolism in muscle, similar to previously reported results in P. vachelli liver (Zhang et al. 2016b). Furthermore, upregulated PDK1, which is member of HIF- $1 \alpha$ target gene, acts to suppress PDHA, involved in inhibiting the initiation of the "TCA cycle". The downregulation of all rate-limiting enzymes of the "TCA cycle" (CS, IDH, DLD, miR-193b/457a/15b-OGDH, miR-210/338-SDHb, SDHa, miR-457a/214-MDH1) indicates an inhibition of aerobic metabolism (Table 1 and Fig. 5 ) (Cerretelli\& Gelfi 2011).

Surprisingly, both the rate-limiting enzymes (PK, miR-181a/-338-HK1, HK2, PFK1) of "Glycolysis/Gluconeogenesis" and LDH were significantly downregulated (Table $\mathbf{1}$ and Fig. $\mathbf{5}$ ), contrary to the accepted metabolic model of hypoxic stress. Such lower level for anaerobic glycolysis was found at both transcriptional and protein levels in $P$. vachelli muscle induced by hypoxia. However, these results were different from that of other fishes. For example, hypoxia increased glycolysis-related enzyme activities and LDH in the $P$. vachelli liver and $D$. rerio muscle, respectively (Chenet al. 2013a). It may be that the muscles in our treatment undergo more severe hypoxia, resulting in a cellular metabolic disorder.

\subsection{Energy metabolism}

(1) Mitochondrial dysfunction

"Oxidative phosphorylation" enables the generation of ATP in the mitochondrial inner membrane and is the main source of energy in eukaryotes. It is generally accepted that hypoxia or ischemia inhibits mitochondrial oxidative phosphorylation and reduces the overall efficiency of energy metabolism(Cerretelli\& Gelfi 2011). We found that all 34 DEP of "Oxidative phosphorylation" were downregulated (Fig. 2), indicating that the coupling reaction of ADP and inorganic phosphate to ATP through the respiratory chain was inhibited to a certain extent. It also reflected that reduced locomotion powered by skeletal muscle might pose a minimal threat to survival under hypoxia when swimming activity will be minimized. 
Meanwhile we found that downregulated expression of the regulator of mitochondrial biogenesis (PPRC1, GFM1, LONP1, SERAC1) and the downstream mitochondrial related functional gene/proteins (COX1/2/4, Mt-Cyb, miR-457a/15b-UQCRC2, UQCRC1, CYC1, Cyb561a3, miR-457a/338/15b/PC-7236-ATP5A1, ATP5B), whichsuggested that mitochondria may be dysfunctional. (Table 1 and Fig. $\mathbf{5}$ ). Such mitochondrial damage induced by hypoxia may lead to the production of ROS and the decline of membrane potential of mitochondria, causing a metabolic disorder and apoptosis (Wang et al. 2019).

(2) Catabolism and anabolism

$P$. vachelli has been shown to have an acute response to hypoxia, including activation of catabolic capacity for more energy, and reduction of biosynthetic capacity to reduce energy expenditure in liver. Similar results are found in P. vachelli muscles, for example, upregulation of PPAR $\alpha / \beta$ is involved in lipid metabolism and lipid oxidation of skeletal muscle in low oxygen environments (A. et al. 2018)(Table 1 ). Also hypoxia induces the activation of riboflavin catabolism through upregulation of RFK as the key enzyme, which improves carbohydrate, lipid, amino acid metabolism in low oxygen environments(Wanget al. 2014). In this study, we also found ample evidence of significantly increased catabolism in $P$. vachelli muscles. The transport-related enriched pathways (e.g., SNARE interactions in vesicular transport, and ABC transporters) and catabolic pathways (e.g., pyrimidine metabolism, central carbon metabolism in cancer) contained a large proportion of upregulated genes (Fig. 2A and 2C ). Furthermore, we found a significant decrease in intermediate metabolites in catabolism in metabolome KEGG analysis (e.g., Metabolic pathways, Glutathione, Purine and Linoleic acid metabolism), which indicated that the consumption of these intermediate metabolites under hypoxia exceeds their synthesis (Fig. 2C ).

Furthermore, the upregulated intermediate metabolites were a big part among anabolic pathways (e.g., biosynthesis of aminoacyl-tRNA, plant hormone, alkaloids derived from histidine, purine, ornithine, lysine and nicotinic acid) in this study (Fig. 2C ). This indicated that consumption of these intermediate metabolites was reduced and accumulated. Additionally, we identified a large portion of downregulated genes/proteins enriched in amino acid biosynthesis pathways (e.g., aminoacyl-tRNA, ribosome, tryptophan, lysine, cysteine, and methionine). These results confirmed the downregulation of anabolism in fish muscle induced by hypoxia, especially in amino acid synthesis (Hardy et al. 2013)(Fig. 2A and 2B ).

\subsection{Muscle function}

Our previous research found that in $P$. vachelli liver and brain tissues, the oxidative stress indices and antioxidant enzymatic activities were activated to varying degrees induced by hypoxia (Zhang et al. 2016a). Similarly, we detected upregulated Mn-SOD and GSTM/T3 mRNAs in P. vachellimuscles (Table 1 ). These might promote the inflammasome activation and trigger the ROS-induced apoptotic pathway (Giraud-Billoud et al. 2019).

\section{(1) Inflammatory response}

Several of the KEGG pathways, enriched by transcriptomes with a large proportion of upregulated genes were associated with human immune (e.g., B cell receptor signaling pathway and Type II diabetes mellitus) and inflammation (e.g., NAFLD, NOD-like receptor and Fc epsilon RI signaling pathway). Of note, the NODlike receptor signaling pathway with 27 upregulated genes may offer insight into the molecular adaptations involved in hypoxia response (Fig. 2A ). NLRs (NLRC3/P3/P12, up-regulated) sense the cytosolic presence of DAMP, mainly from mitochondria (e.g., ROS and mtDNA). Then NLRs drove the activation of NFkappa-B (IFNGR1/IKBKG/NFKBIB/CHUK, up-regulated) and MAPK (MAPKAPK5/Map4k2/Map2k4, up-regulated), cytokine production and apoptosis (Chen et al. 2014; Ko et al. 2017). Alternatively, a

different set of NLRs induced caspase-1 activation through the assembly of multiprotein complexes called inflammasomes. The activated of caspase-1 regulates maturation of the pro-inflammatory cytokines IL-13and drives mitochondrial dysfunction and apoptosis (Liu et al. 2018; Yi et al. 2015)(Table 1 ).

(2) Apoptosis

Studies have shown that the apoptosis of fish brain and cardiac cells in the hypoxic state is one of the main 
causes of "pond turnover" (Xiao 2015). Researchers have detected caspase-3/9 or apoptotic index, and found that hypoxia stress can induce apoptosis of fish neural, liver or cardiac cells, e.g., Acipenser shrenckii (Lu et al. 2005),Gymnocephalus cernua, Platichthys flesus(Tiedke et al. 2014) and M. amblycephala (Sun et al. 2017). We first found that there was a significant cell cycle arrest (CDK10/12/13/CCNI/YWHAZ, MCM4/7 down-regulated, CCNG2/DIDO1/MDC1/CDKN1B/GADD45A up-regulated) in $P$. vachellimuscles under hypoxia (Table 1 ), which may be due to the blocked energy metabolism and inflammatory response (Skovira et al. 2016). C-fos and HMGB1/3 were often the marker of cell apoptosis, and plays a significant role in hypoxia or ischemia induced apoptosis, which were significantly upregulated in $P$. vachelli muscle (Fig.6 ) (Brunelle\& Chandel 2002). "Apoptosis" was also significantly enriched in KEGG pathway enrichment analysis of miRNA-mRNA pairs( Fig. 3A).

Fig. 6. DEG/DEP enrichment of apoptotic pathways including extrinsic, mitochondrial, endoplasmic reticulum stress, and relative mRNA expression of 22 selected DEG for apoptosis, with respect to mRNA-seq and qRT-PCR. For abbreviations and explanations, please see the text.

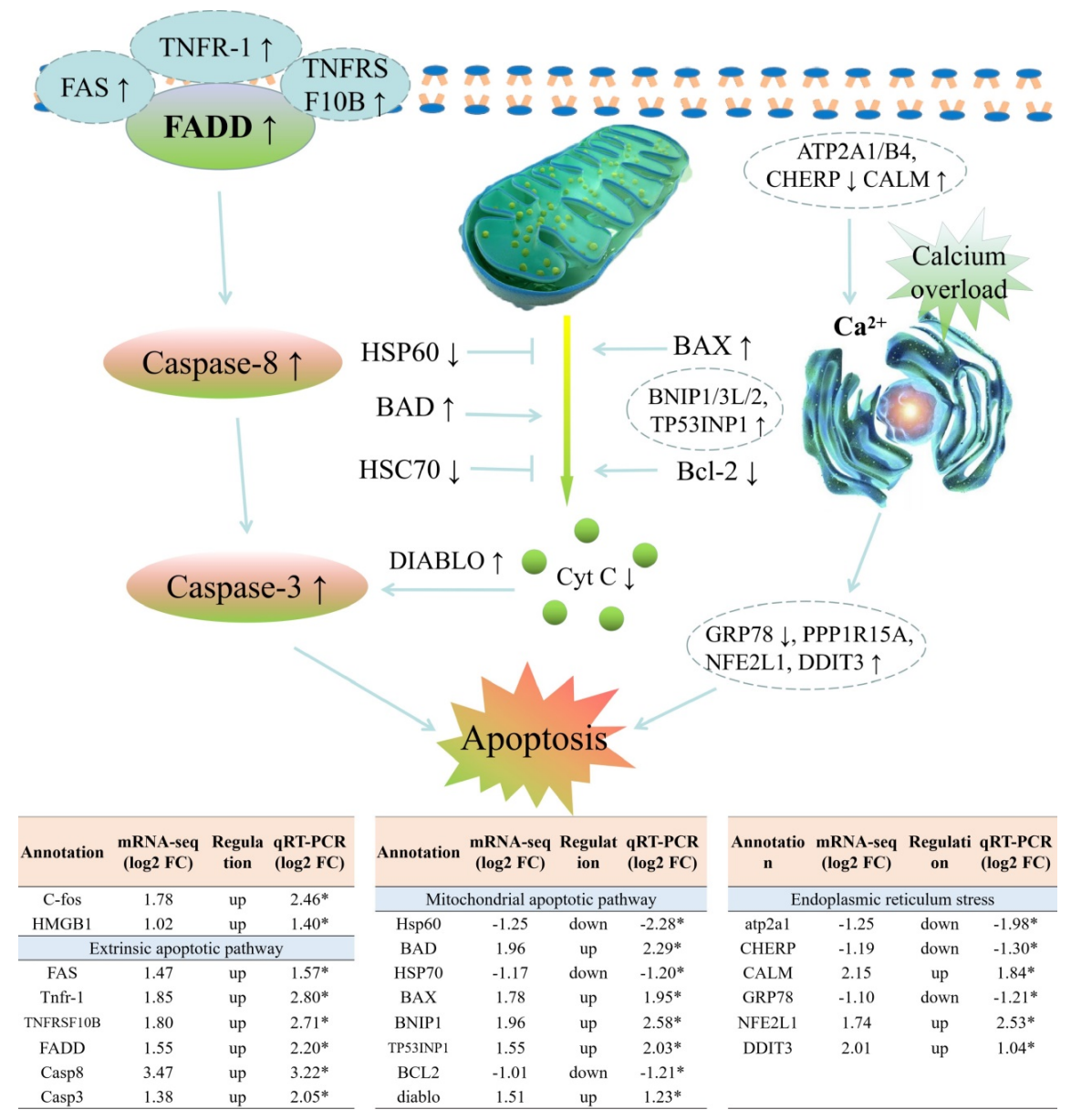

At last the three paths of apoptosis (extrinsic, mitochondrial, and endoplasmic reticulum stress) were enriched by abundant DEG/DEP (Fig. 6 ). For instance in 145 negative miRNA-mRNA-protein pairs, Hadha was downregulated and promoted cell apoptosis by decreasing the acylation of monolysocardiolipin into cardiolipin via raised miR-1-2 (Taylor et al. 2012). And that GRP78 is expressed in large quantities under low oxygen to maintain the stability of the endoplasmic reticulum and protect cells (Reddyet al. 2003). Upregulation of miR-457a led to endoplasmic reticulum stress and hypoxia-induced apoptosis by targeting 
GRP78 in P. vachelli muscles (Table 1 and Fig. 5 ).

(3) Muscle dysfunction

Several of the KEGG enriched by multi-omics $(P<0.05)$ were associated with impaired contraction or dilation of heart muscle (DCM and HCM) and dyskinesia (Parkinson's and Huntington's disease), which suggested that hypoxia induces muscle dysfunction (Fig. 2 and Fig. 3A ). In addition, "Vascular smooth muscle contraction" and "Calcium signaling pathway" contained abnormal muscular and calcium overload related proteins that were significantly enriched by down DEP. For example, Atp2a1, a key regulator of muscle performance, contributed to calcium sequestration involved in muscular excitation/contraction (Odermattet al. 2000). Krt8 helps to link the contractile apparatus to dystrophin at the costamere of striated muscle (Ursitti et al. 2004). In our results (Table 1 and Fig. 5 ), let-7b was upregulated, whereas its target genes/proteins Atp2a1 and Krt8 were significantly downregulated. This supported the idea that calcium overload and muscle dysfunction were linked in hypoxia adaptation in $P$. vachelli .

To date, understanding of small RNAs in fishes is limited and informations regarding hypoxic miRNA markers are especially rare. Compared with other animals or cell models, 24 miRNAs reported previously as hypoxia-responsive were identified (e.g., miR-15b, 133b, 143, 146b, 181a, 193b, 27b, 301b, 338, 152, 18a, 30a/d, 126, 199a, 210, 214, 29b, 25, let-7b) from 39 DEMI in our results. These are thought to exert broad pleiotropic effects by targeting genes involved in cell cycle arrest, metabolism, apoptosis, cell survival and mitochondrial function (Bandara et al. 2017; Guimbellot et al. 2009; McCormick et al. 2010; Pocock 2011). Additionally, in fishes, the tendencies of miR-210, -193b, -181a expression among muscle were the same as the liver of $P$. vachelli(Zhang et al. 2016b) or M. amblycephala (Sun et al. 2017) . For example, in negative miRNA-mRNA-protein pairs (Table S4 ), several key enzymes of "TCA cycle", e.g., miR-193bOGDH and miR-210-SDHb/SUCLA2, were detected in our conjoint analysis. Moreover, the same result as Danio rerio that raised let-7b acts downstream of HIF- $1 \alpha$ to repress cell proliferation through blocking cell cycle progression (Huang et al. 2017). Interestingly, several miRNA expressions in P. vachelli muscle were the opposite to that of liver (e.g., miR-27b, -143, -338), which may be due to different tissues or a metabolic disorder in muscle induced by hypoxia. For instance, data analysis of predicted multi-omics pairsin silico (Table S4), suggested that the possible mechanism that GAPVD1, a protein required for efficient endocytosis (Guillen et al. 2020), promoted catabolism in muscles under hypoxia by downregulating miR-27b/143. Furthermore, upregulating miR-338 negatively targeted key enzymes of glycolysis (HK1) and "oxidative phosphorylation" (SDH, Atp5a1, NDUFV1/S1) under hypoxic conditions in P. vachelli muscle (Aschrafi et al. 2012).

Fig. 7. A schematic model of acute reactions inPelteobagrus vachelli muscles caused by acute hypoxia. For abbreviations and explanations, please see the text.

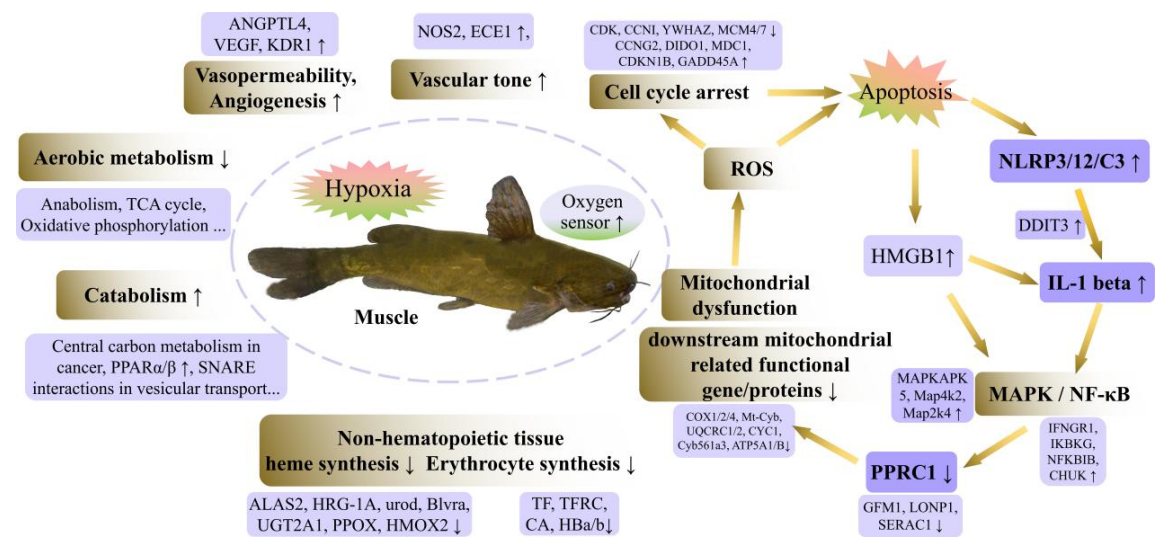

\section{Conclusions}


We identified a large number of hypoxia associated markers in the muscles of $P$. vachelli that were involved in diverse biological pathways such as HIF-1 signaling, energy metabolism, and muscle function. Compared with the $P$. vachelli livers, we found that muscles experienced significant hypoxia-associated changes, and this was evident in discrete tissue-specific patterns present. For example, P. vachelli livers have increased anaerobic glycolysis, heme synthesis, erythropoiesis, and inhibited apoptosis when exposed to under $4 \mathrm{~h}$ of hypoxia, whereas the opposite was true to muscles.

To maintain normal physical activity, fishes acutely react to acute hypoxia, by activated catabolic pathways to generate more energy, decreased biosynthesis to reduce energy consumption, and shifted from aerobic to anaerobic metabolic contributions. We found that hypoxia induced muscle dysfunction through impairing mitochondrial function, activating inflammasome, and apoptosis (Zhou et al. 2011). The hypoxia-induced mitochondrial dysfunction enhanced ROS generation and apoptosis, further triggering IL-1 $\beta$ production via the inflammasome activation (Pomerantz et al.2001). In turn, IL-1 $\beta$ further impaired mitochondrial function and apoptosis by suppressing downstream mitochondrial biosynthesis related proteins, resulting in a vicious circle between inflammasome activation and mitochondrial dysfunction (Yi et al. 2015) (Fig. 7 ). We hope that it provides fundamental data on analytical methods, as well as biological and mechanical insights into future research for environmental stress induced hypoxia.

Fig. S1 Hierarchical clustering of DEG, DEMI, and DEP across six libraries and neg-DEM, and pos-DEM across 20 samples

Table S1. Primer descriptions

Table S2. Summary of all genes, miRNAs, proteins, and metabolites

Table S3. Quality control analysis of transcriptome, miRNAome, proteome, and metabolome

Table S4. List of miRNA-mRNA and miRNA-mRNA-protein pairs with negative correlation

Table S5. Multi-omics identification of key miRNAs, genes, proteins, and metabolites

\section{Abbreviations}

VEGF, vascular endothelial growth factor A; qRT-PCR, quantitative real-time PCR; NGST, next-generation sequencing technologies; 2D-DIGE, two-dimensional fluorescence difference gel electrophoresis; iTRAQ, Isobaric tag for relative and absolute quantitation; TMT, Tandem Mass Tags; MS, mass spectrometry; KEGG, Kyoto Encyclopedia of Genes and Genomes; DEG, differentially expressed genes; DEMI, differentially expressed miRNAs; DEP, differentially expressed proteins; VIP, variable importance in projection; DEM, differentially expressed metabolites; LC-ESI-MS/MS, liquid chromatography-electrospray ionization-tandem mass spectrometry; DCM, Dilated cardiomyopathy (DCM); HCM, Hypertrophic cardiomyopathy (HCM); PLS-DA , Partial least-squares-discriminant analysis; PHD2, egl nine homolog 1; PHD3, egl nine homolog 3; FIH-1, Hypoxia-inducible factor 1-alpha inhibitor; KDR1, vascular endothelial growth factor receptor kdr; ANGPTL4, angiopoietin-related protein 4; NOS2, nitric oxide synthase, inducible; ECE1, endothelinconverting enzyme 1; ALAS2, 5-aminolevulinate synthase, erythroid-specific, mitochondrial; HRG-1A, heme transporter hrg1-A; urod, uroporphyrinogen decarboxylase; Blvra, Biliverdin reductase A; UGT2A1; UDPglucuronosyltransferase 2A1; PPOX, protoporphyrinogen oxidase; HMOX2, Heme oxygenase 2; TF, transferrin; TFRC, transferrin receptor protein 1; CA, carbonic anhydrase; $\mathrm{HB} \alpha / \beta$, Hemoglobin subunit alpha/beta; GLUT1, Solute carrier family 2, facilitated glucose transporter member 1; PK, pyruvate kinase; HK, hexokinase; PFK1, 6-phosphofructokinase; LDH, L-lactate dehydrogenase; PDK, pyruvate dehydrogenase kinase isoform 1; PDHA, Pyruvate dehydrogenase; CS, Citrate synthase, mitochondrial; IDH, Isocitrate dehydrogenase [NADP], mitochondrial; DLD, Dihydrolipoyl dehydrogenase, mitochondrial; OGDH, 2-oxoglutarate dehydrogenase, mitochondrial; sdha/b, Succinate dehydrogenase [ubiquinone] flavoprotein/iron-sulfur subunit, mitochondrial; MDH1, Malate dehydrogenase, cytoplasmic; PPRC1, peroxisome proliferator-activated receptor gamma coactivator-related protein 1; GFM1, elongation factor G, mitochondrial; LONP1, lon protease homolog, mitochondrial; SERAC1, protein SERAC1; COX, cytochrome c oxidase; Mt-Cyb, Cytochrome b; UQCRC1/2, Cytochrome b-c1 complex subunit 1/2, mitochondrial; CYC1, Cytochrome c1, 
heme protein, mitochondrial; Cyb561a3, Cytochrome b ascorbate-dependent protein 3; ATP5A1/B, ATP synthase subunit alpha/beta, mitochondrial; Mn-SOD, manganese superoxide dismutase; GSTM/T3, Glutathione S-transferase Mu 3/theta-3; NLRC3, NLR family CARD domain-containing protein 3; NLRP3/P12, NACHT, LRR and PYD domains-containing protein 3/12; IL-1 $\beta$, Interleukin-1 beta; IFNGR1, interferon gamma receptor 1; IKBKG, NF-kappa-B essential modulator; NFKBIB, NF-kappa-B inhibitor beta; CHUK, inhibitor of nuclear factor kappa-B kinase subunit alpha; MAPKAPK5, MAP kinase-activated protein kinase 5; Map4k2, mitogen-activated protein kinase kinase kinase kinase 2; Map2k4, dual specificity mitogenactivated protein kinase kinase 4; CDK, Cyclin-dependent kinase CCNI, Cyclin-I; YWHAZ, 14-3-3 protein zeta/delta; MCM4/7, DNA replication licensing factor MCM4/7; CCNG2, Cyclin-G2; DIDO1, death-inducer obliterator 1; MDC1, mediator of DNA damage checkpoint protein 1; CDKN1B, cyclin-dependent kinase inhibitor 1B; GADD45A, growth arrest and DNA damage-inducible protein GADD45 alpha; PPAR $\alpha / \beta$, Peroxisome proliferator-activated receptor alpha/delta; C-fos, Proto-oncogene c-Fos; HMGB1/3, High mobility group protein B1/3; FAS, tumor necrosis factor receptor superfamily member 6 ; TNFR1, tumor necrosis factor receptor superfamily member 1A; TNFRSF10B, tumor necrosis factor receptor superfamily member 10B; FADD, FAS-associated death domain protein; Casp3/8, caspase-3/8; HSP60, 60 kDa heat shock protein, mitochondrial; BAD, bcl2-associated agonist of cell death; HSC70, heat shock cognate $70 \mathrm{kDa}$ protein; BAX, apoptosis regulator BAX; BNIP1/2/3L, BCL2/adenovirus E1B $19 \mathrm{kDa}$ protein-interacting protein 1/2/3-like; BCL2, apoptosis regulator Bcl-2; DIABLO, diablo homolog, mitochondrial; ATP2A1, Sarcoplasmic/endoplasmic reticulum calcium ATPase 1; ATP2B4, plasma membrane calcium-transporting ATPase 4; GRP78, 78 kDa glucose-regulated protein; PPP1R15A, protein phosphatase 1 regulatory subunit 15B; NFE2L1, nuclear factor erythroid 2-related factor 1; DDIT3, DNA damage-inducible transcript 3 protein

\section{Ethic}

Our experiments were performed in accordance with Guidelines for the Care and Use of Laboratory Animals in China. This study was approved by NJNU Animal Ethics Committee for Grant No. SYXK (Jiangsu) 2015-0028.

\section{Acknowledgements}

This study was supported by Natural Science Foundation of Shandong Province of China (ZR2019QC002), Key Laboratory of Healthy Freshwater Aquaculture, Ministry of Agriculture and Rural affairs; Key Laboratory of Freshwater Aquaculture genetic and breeding of Zhejiang Province; Zhejiang Institute of Freshwater Fisheries, Huzhou (ZJK202003), Agricultural Science and Technology independent innovation project (CX(19)2034), Creation Project of Major New Species of Agriculture of Jiangsu Province(PZCZ201742) and Qingchuang science and technology support program of Shandong provincial college.

\section{Data Accessibility and Benefit-Sharing Statement}

All data generated or analysed during this study are included in this published article and its supplementary information files. The raw data from the high-throughput sequencing data were deposited at the NCBI Sequence Read Archive (ID: SUB7714154 and SUB7765255). The mass spectrometry proteomics data have been deposited to the ProteomeXchange Consortium via the iProX partner repository with the dataset identifier PXD020425. Metabolome data related to this study has been publicly available on Metabolight (ID: MTBLS1888).The authors declare that they have no conflict of interest.

\section{Author Contributions}

Guosong Zhang, Jie Li: Data curation, Writing- Original draft preparation; Jiejie Xu, Xia Liang, Jiajia Zhang, Kai Zhang, Jie Ji, Tao Wang, Yongyi Jia: Software, Validation, Experiments, Data query; Shaowu Yin: Experimental design, revisions. The authors declare that they have no conflict of interest.

\section{References}

A. OBK, A. HJ, Jules D, et al.(2018) PPAR $\alpha$-independent effects of nitrate supplementation on skeletal muscle metabolism in hypoxia. 1865, S092544391830276X-. Aschrafi A, Kar AN, Natera-Naranjo O, et al. 
(2012) MicroRNA-338 regulates the axonal expression of multiple nuclear-encoded mitochondrial mRNAs encoding subunits of the oxidative phosphorylation machinery. Cell Mol Life Sci69, 4017-4027. Bandara KV, Michael MZ, Gleadle JM (2017) MicroRNA Biogenesis in Hypoxia. Microrna 6, 80-96. Baptista RB, Souza-Castro N, Almeida-Val VM (2016) Acute hypoxia up-regulates HIF-1alpha and VEGF mRNA levels in Amazon hypoxia-tolerant Oscar (Astronotus ocellatus).Fish Physiol Biochem 42, 1307-1318. Beck BH, Fuller SA, Li C, et al. (2016) Hepatic transcriptomic and metabolic responses of hybrid striped bass (Morone saxatilisxMorone chrysops) to acute and chronic hypoxic insult. Comp Biochem Physiol Part D Genomics Proteomics 18, 1-9. Brunelle JK, Chandel NS (2002) Oxygen deprivation induced cell death: an update. Apoptosis 7, 475-482. Cao M, Li C, Liu Y, Cai K, Zhou X (2020) Assessing Urinary Metabolomics in Giant Pandas Using Chromatography/Mass Spectrometry: Pregnancy-Related Changes in the Metabolome. Frontiers in Endocrinology 11. Cerretelli P, Gelfi C (2011) Energy metabolism in hypoxia: reinterpreting some features of muscle physiology on molecular grounds. European Journal of Applied Physiology111, p.421-432. Chen K, Cole RB, Rees BB (2013a) Hypoxia-induced changes in the zebrafish (Danio rerio) skeletal muscle proteome. J Proteomics 78, 477-485. Chen N, Chen LP, Zhang J, et al. (2012) Molecular characterization and expression analysis of three hypoxia-inducible factor alpha subunits, HIF- $1 \alpha / 2 \alpha / 3 \alpha$ of the hypoxia-sensitive freshwater species, Chinese sucker. Gene498, 0-90. Chen Z, Liu Y, Sun B, et al.(2014) Polyhydroxylated metallofullerenols stimulate IL-1beta secretion of macrophage through TLRs/MyD88/NFkappaB pathway and NLRP(3) inflammasome activation. Small 10, 2362-2372. Chen Z, Wen B, Wang Q, et al.(2013b) Quantitative proteomics reveals the temperature-dependent proteins encoded by a series of cluster genes in thermoanaerobacter tengcongensis. Mol Cell Proteomics 12, 2266-2277. Dowd WW, Renshaw GM, Cech JJ, Jr., Kultz D (2010) Compensatory proteome adjustments imply tissue-specific structural and metabolic reorganization following episodic hypoxia or anoxia in the epaulette shark (Hemiscyllium ocellatum). Physiol Genomics 42, 93-114. Everett MV, Antal CE, Crawford DL (2012) The effect of short-term hypoxic exposure on metabolic gene expression. J Exp Zool A Ecol Genet Physiol 317, 9-23. Geng X, Feng J, Liu S, et al.(2014) Transcriptional regulation of hypoxia inducible factors alpha (HIF-alpha) and their inhibiting factor (FIH-1) of channel catfish (Ictalurus punctatus) under hypoxia. Comp Biochem Physiol B Biochem Mol Biol 169, 38-50. Giraud-Billoud M, Rivera-Ingraham GA, Moreira DC, et al. (2019) Twenty years of the 'Preparation for Oxidative Stress' (POS) theory: Ecophysiological advantages and molecular strategies. Comp Biochem Physiol A Mol Integr Physiol234, 36-49. Gong D, Xu L, Li W, Shang R, Liu S (2020) Comparative analysis of liver transcriptomes associated with hypoxia tolerance in the gynogenetic blunt snout bream.Aquaculture, 735163. Gracey AY, Troll JV, Somero GN (2001) Hypoxia-induced gene expression profiling in the euryoxic fish Gillichthys mirabilis. Proc Natl Acad Sci U S A 98, 1993-1998. Guillen RX, Beckley JR, Chen J-S, Gould KL (2020) CRISPR-mediated gene targeting of CK1 $\delta / \varepsilon$ leads to enhanced understanding of their role in endocytosis via phosphoregulation of GAPVD1. entific Reports 10. Guimbellot JS, Erickson SW, Mehta T, et al. (2009) Correlation of microRNA levels during hypoxia with predicted target mRNAs through genome-wide microarray analysis.BMC Med Genomics 2, 15. Guosong, Zhang, Cheng, et al.(2017) Identification of HIF-1 signaling pathway in Pelteobagrus vachelli using RNASeq: effects of acute hypoxia and reoxygenation on oxygen sensors, respiratory metabolism, and hematology indices. Journal of Comparative Physiology B Biochemical Systemic \& Environmental Physiology. Hardy KM, Burnett KG, Burnett LE (2013) Effect of hypercapnic hypoxia and bacterial infection (Vibrio campbellii) on protein synthesis rates in the Pacific whiteleg shrimp, Litopenaeus vannamei. Am J Physiol Regul Integr Comp Physiol305, R1356-1366. Huang CX, Chen N, Wu XJ, et al. (2017) Zebrafish let-7b acts downstream of hypoxia-inducible factor-1alpha to assist in hypoxia-mediated cell proliferation and cell cycle regulation. Life Sci 171, 21-29. Ju Z, Wells MC, Heater SJ, Walter RB (2007) Multiple tissue gene expression analyses in Japanese medaka (Oryzias latipes) exposed to hypoxia. Comp Biochem Physiol C Toxicol Pharmacol 145, 134-144. Ko JH, Yoon SO, Lee HJ, Oh JY (2017) Rapamycin regulates macrophage activation by inhibiting NLRP3 inflammasome-p38 MAPK-NFkappaB pathways in autophagy- and p62-dependent manners. Oncotarget 8, 40817-40831. Lardon I, Eyckmans M, Vu TN, et al. (2013) 1H-NMR study of the metabolome of a moderately hypoxia-tolerant fish, the common carp (Cyprinus carpio).Metabolomics 9, 1216-1227. Li W, Yang Y, Liu Y, et al.(2017) Integrated analysis of mRNA and miRNA expression profiles in livers of Yimeng black pigs with extreme phenotypes for backfat thickness. Oncotarget 8, 114787-114800. Liu Q, Zhang D, 
Hu D, Zhou X, Zhou Y (2018) The role of mitochondria in NLRP3 inflammasome activation. Mol Immunol 103, 115-124. Lu G, Mak YT, Wai SM, et al.(2005) Hypoxia-induced differential apoptosis in the central nervous system of the sturgeon (Acipenser shrenckii). Microsc Res Tech68, 258-263. Mandic M, Ramon ML, Gracey AY, Richards JG (2015) Divergent transcriptional patterns are related to differences in hypoxia tolerance between the intertidal and the subtidal sculpins. Molecular Ecology 23, 6091-6103. McCormick R, Buffa FM, Ragoussis J, Harris AL (2010) The role of hypoxia regulated microRNAs in cancer.Curr Top Microbiol Immunol 345, 47-70. Mortazavi A, Williams BA, McCue K, Schaeffer L, Wold B (2008) Mapping and quantifying mammalian transcriptomes by RNA-Seq. Nat Methods 5, 621-628. Odermatt A, Barton K, Khanna VK, et al. (2000) The mutation of Pro789 to Leu reduces the activity of the fast-twitch skeletal muscle sarco(endo)plasmic reticulum Ca2+ ATPase (SERCA1) and is associated with Brody disease. Human Genetics 106, 482-491. Patro R, Duggal G, Love MI, Irizarry RA, Kingsford C (2017) Salmon provides fast and bias-aware quantification of transcript expression. Nat Methods 14, 417-419. Pocock R (2011) Invited review: decoding the microRNA response to hypoxia. Pflugers Arch461, 307-315. Pomerantz, Benjamin, J., et al. (2001) Inhibition of caspase 1 reduces human myocardial ischemic dysfunction via inhibition of IL-18 and. Proc Natl Acad Sci U S A. Reddy RK, Mao C, Baumeister P, et al. (2003) Endoplasmic reticulum chaperone protein GRP78 protects cells from apoptosis induced by topoisomerase inhibitors: role of ATP binding site in suppression of caspase-7 activation. J Biol Chem 278, 20915-20924. Robinson MD, McCarthy DJ, Smyth GK (2010) edgeR: a Bioconductor package for differential expression analysis of digital gene expression data. Bioinformatics 26, 139-140. Sappal R, Fast M, Purcell S, et al. (2016) Copper and hypoxia modulate transcriptional and mitochondrial functional-biochemical responses in warm acclimated rainbow trout (Oncorhynchus mykiss). Environ Pollut 211, 291-306. Skovira JW, Wu J, Matyas JJ, et al. (2016) Cell cycle inhibition reduces inflammatory responses, neuronal loss, and cognitive deficits induced by hypobaria exposure following traumatic brain injury. $J$ Neuroinflammation 13, 299. Smith RW, Cash P, Ellefsen S, Nilsson GE (2009) Proteomic changes in the crucian carp brain during exposure to anoxia. Proteomics 9, 2217-2229. Sun S, Guo Z, Fu H, Zhu J, Ge X (2018) Integrated metabolomic and transcriptomic analysis of brain energy metabolism in the male Oriental river prawn (Macrobrachium nipponense) in response to hypoxia and reoxygenation. Environ Pollut 243, 1154-1165. Sun S, Xuan F, Ge X, Zhu J, Zhang W (2017) Dynamic mRNA and miRNA expression analysis in response to hypoxia and reoxygenation in the blunt snout bream (Megalobrama amblycephala).Sci Rep 7, 12846. Taylor WA, Mejia EM, Mitchell RW, et al. (2012) Human trifunctional protein alpha links cardiolipin remodeling to beta-oxidation. PLoS One 7, e48628. Tiedke J, Thiel R, Burmester T (2014) Molecular response of estuarine fish to hypoxia: a comparative study with ruffe and flounder from field and laboratory. PLoS One9, e90778. Ursitti JA, Lee PC, Resneck WG, et al. (2004) Cloning and characterization of cytokeratins 8 and 19 in adult rat striated muscle. Interaction with the dystrophin glycoprotein complex. J Biol Chem 279, 41830-41838. Wang D, Du Y, Xu H, Pan H, Wang R (2019) Paeonol protects mitochondrial injury and prevents pulmonary vascular remodeling in hypoxia. Respir Physiol Neurobiol268, 103252. Wang H, Huang C, Chen N, et al. (2015) Molecular characterization and mRNA expression of HIF-prolyl hydroxylase-2 (phd2) in hypoxia-sensing pathways from Megalobrama amblycephala. Comp Biochem Physiol B Biochem Mol Biol186, 28-35. Wang YP, Wei JY, Yang JJ, et al. (2014) Riboflavin supplementation improves energy metabolism in mice exposed to acute hypoxia. Physiol Res 63, 341-350. Wen X, Hu Y, Zhang X, Wei X, Yin S (2019) Integrated application of multi-omics provides insights into cold stress responses in pufferfish Takifugu fasciatus. BMC Genomics 20. Wulff T, Jokumsen A, Hojrup P, Jessen F (2012) Time-dependent changes in protein expression in rainbow trout muscle following hypoxia. J Proteomics 75, 2342-2351. Xiao W (2015) The hypoxia signaling pathway and hypoxic adaptation in fishes. Sci China Life Sci58, 148-155. Yang Y, Fu Q, Wang X, et al.(2018) Comparative transcriptome analysis of the swimbladder reveals expression signatures in response to low oxygen stress in channel catfish, Ictalurus punctatus. Physiol Genomics 50, 636-647. Yi W, Yiran L, Bicheng L (2015) VICIOUS CIRCLE OF NLRP3 AND MITOCHONDRIAL DAMAGE PLAYS CENTRAL ROLE IN RENAL ISCHEMIA REPERFUSION INJURY. Nephrology Dialysis Transplantation 30, iii3-iii4. Zhang G, Li J, Zhang J, et al.(2019) Integrated Analysis of Transcriptomic, miRNA and Proteomic Changes of a Novel Hybrid Yellow Catfish Uncovers Key Roles for miRNAs in Heterosis. Mol Cell Proteomics 18, 1437-1453. Zhang G, Mao J, Liang F, et al. (2016a) Modulated expression and enzymatic activities of 
Darkbarbel catfish, Pelteobagrus vachelli for oxidative stress induced by acute hypoxia and reoxygenation. Chemosphere 151, 271-279. Zhang G, Yin S, Mao J, et al.(2016b) Integrated analysis of mRNA-seq and miRNA-seq in the liver of Pelteobagrus vachelli in response to hypoxia. Sci Rep 6, 22907. Zhang G, Zhang J, Wen X, et al. (2017) Comparative iTRAQ-Based Quantitative Proteomic Analysis of Pelteobagrus vachelli Liver under Acute Hypoxia: Implications in Metabolic Responses. Proteomics 17. Zhao C, Zhang G, Yin $\mathrm{S}$, et al.(2017) Integrated analysis of mRNA-seq and miRNA-seq reveals the potential roles of sex-biased miRNA-mRNA pairs in gonad tissue of dark sleeper (Odontobutis potamophila). BMC Genomics 18, 613. Zhong XP, Dan W, Zhang YB, Gui JF (2009) Identification and characterization of hypoxia-induced genes in Carassius auratus blastulae embryonic cells using suppression subtractive hybridization. Comparative Biochemistry 83 Physiology Part B Biochemistry $\&$ Molecular Biology 152, 0-170. Zhou R, Yazdi AS, Menu P, Tschopp J (2011) A role for mitochondria in NLRP3 inflammasome activation. Nature 469, 221-225. Zhu CD, Wang ZH, Yan B (2013) Strategies for hypoxia adaptation in fish species: a review. J Comp Physiol B 183, 1005-1013. 